\title{
Retraction Note to: Urinary parameters predictive and electrolyte disturbances of cisplatin-induced acute renal associated with cancer as a critical target of the chemotherapeutic agent in patients with solid tumors
}

\author{
Behnam Pedram $^{1}$ - Ahmad Taghavi Moghadam ${ }^{2}$ - Zahra Kamyabi-Moghaddam ${ }^{3,9}$ • \\ Omid Mavedati ${ }^{4}$ Babak Abbas Beigi ${ }^{5}$ - Adel Khodaei Sharabiyani ${ }^{6}$. \\ Ali Bashiri Dezfuli ${ }^{7}$ - Soheil Khalili ${ }^{6}$ - Ali Mohammad Bahrami ${ }^{8}$ - Alireza Nasoori ${ }^{6}$
}

Published online: 5 November 2016

(C) International Society of Oncology and BioMarkers (ISOBM) 2016

\section{Retraction Note to: Tumor Biol (2015) 36:4495-4500 \\ DOI 10.1007/s13277-015-3091-1}

This article has been retracted at the request of the Editorin-Chief, the International Society of Oncology and BioMarkers (ISOBM) and the Publisher per the Committee on Publication Ethics guidelines. The article shows evidence of irregularities in authorship during the submission process and there is strong reason to believe that the peer review process was compromised.

As such the validity of the content of this article cannot be verified.

The online version of the original article can be at http://dx.doi. org/10.1007/s13277-015-3091-1.

Ali Mohammad Bahrami

am.bahrami@ilam.ac.ir

1 Department of Pathobiology, Susangerd Branch, Islamic Azad University, Susangerd, Iran

2 Razi Vaccine and Serum Research Institute, Ahwaz Branch, Ahwaz, Iran

3 Department of Medicine II, Klinikum rechts der Isar, TU Muenchen, Munich, Germany

4 Department of Clinical Science, Faculty of Veterinary Medicine, University of Tehran, Tehran, Iran
5 Department of Medical History, Mazandaran University of Medical Sciences, Sari, Iran

6 Graduate of Faculty of VeterinaryMedicine, Islamic Azad University, Karaj Branch, Rajaee Shahr, Moazen Blvd., Karaj, Iran

7 Department of Toxicology, Faculty of Veterinary Medicine, University of Tehran, Tehran, Iran

8 Faculty of Para Veterinary Medicine, Ilam University, Ilam, Iran

9 Department of Pathology, Faculty of Veterinary Medicines, Tehran University, Tehran, Iran 